Investitionsrechnung für Praktiker 
Bernd Heesen

\section{Investitionsrechnung für Praktiker}

Fallorientierte Darstellung der Verfahren und Berechnungen

3. Auflage

Springer Gabler 


\section{Bernd Heesen \\ Marktschellenberg \\ Deutschland}

Die Darstellung von manchen Formeln und Strukturelementen war in einigen elektronischen Ausgaben nicht korrekt, dies ist nun korrigiert. Wir bitten damit verbundene Unannehmlichkeiten zu entschuldigen und danken den Lesern für Hinweise.

ISBN 978-3-658-10355-2

ISBN 978-3-658-10356-9 (eBook)

DOI 10.1007/978-3-658-10356-9

Die Deutsche Nationalbibliothek verzeichnet diese Publikation in der Deutschen Nationalbibliografie; detaillierte bibliografische Daten sind im Internet über http://dnb.d-nb.de abrufbar.

\section{Springer Gabler}

(C) Springer Fachmedien Wiesbaden 2016

Das Werk einschließlich aller seiner Teile ist urheberrechtlich geschützt. Jede Verwertung, die nicht ausdrücklich vom Urheberrechtsgesetz zugelassen ist, bedarf der vorherigen Zustimmung des Verlags. Das gilt insbesondere für Vervielfältigungen, Bearbeitungen, Übersetzungen, Mikroverfilmungen und die Einspeicherung und Verarbeitung in elektronischen Systemen.

Die Wiedergabe von Gebrauchsnamen, Handelsnamen, Warenbezeichnungen usw. in diesem Werk berechtigt auch ohne besondere Kennzeichnung nicht zu der Annahme, dass solche Namen im Sinne der Warenzeichenund Markenschutz-Gesetzgebung als frei zu betrachten wären und daher von jedermann benutzt werden dürften. Der Verlag, die Autoren und die Herausgeber gehen davon aus, dass die Angaben und Informationen in diesem Werk zum Zeitpunkt der Veröffentlichung vollständig und korrekt sind. Weder der Verlag noch die Autoren oder die Herausgeber übernehmen, ausdrücklich oder implizit, Gewähr für den Inhalt des Werkes, etwaige Fehler oder Äußerungen.

Lektorat: Andreas Funk

Einbandentwurf: KünkelLopka GmbH, Heidelberg

Gedruckt auf säurefreiem und chlorfrei gebleichtem Papier 


\section{Vorwort}

Dieses Buch ist für Praktiker im Studium und im Beruf, die Investitionen planen, begleiten und überprüfen müssen. Es ist aber auch ein Buch für alle diejenigen, die über Finanzierungen von Investitionen reden, diese verhandeln und genehmigen und damit geplante Investitionen generell präsentieren müssen.

Es ist hingegen kein trockenes Lehrbuch, das akademisch geprägt möglichst viele Facetten abbilden möchte. Vollständigkeit im wissenschaftlichen Sinn ist nicht Ziel dieses Buches.

,Für Praktiker' heißt aber auch nicht, dass hier versucht wird, die mathematische Basis und das Verständnis um betriebswirtschaftliche Zusammenhänge möglichst einfach zu halten bzw. teilweise auszublenden. ,Für Praktiker ${ }^{6}$ heißt, dass das Wesentliche und die wichtigsten Ansätze im Detail anhand eines durchgehenden konkreten Excel basierten Berechnungsbeispiels durchgesprochen bzw. durchgearbeitet werden.

Wir erarbeiten uns somit im Buch eine komplette mehrperiodische Investitionsrechnung, die aus verschiedenen Blickwinkeln heraus und mit unterschiedlichen Ansätzen ausgewertet wird. Die Investition wird auch in eine für Banken wichtige Gewinn- und Verlustrechnung überführt.

Dafür sind auch mathematische Zusammenhänge von Bedeutung und die Formeln sehen auf den ersten Blick nicht immer einladend aus. Aber, wir brechen diese Formeln auf, zerlegen Sie also und gehen Schritt für Schritt vor, immer auch mit den Zahlen aus unserem Excel basierten Beispiel.

Das genannte Excel Tool können Sie sich aus dem Internet entweder auf der Seite von Springer Gabler (www.springer.com) oder auf der Homepage meiner Akademie (www. ifak-bgl.com) kostenfrei herunterladen. Es ist dort in zwei Versionen erhältlich:

- eine Übungsversion, mit der Sie selbst alle Rechen- und Analyseschritte parallel zur Lektüre des Buches aufbauen und nachrechnen können

- eine fertige Version, in der Sie nur „Ihre“ Werte eingeben müssen und Sie dann sofort alle besprochenen Auswertungen sofort ohne weitere Eigenarbeiten berechnet bekommen. 
Zum besseren Verständnis sollten Sie aber parallel zum Lesen selbst mit dem Rechentool in der Übungsvariante arbeiten. Es hilft ungemein beim Verständnis und macht Spaß.

Haben Sie keine Lust auf oder keinen Zugang zu Excel? Das macht trotzdem nichts. Alle Analysen und Berechnungen sowie die Ergebnisse werden sukzessiv besprochen und mit den entsprechenden Originaltabellen und Grafiken (aus dem Excel Tool) dargestellt.

,Durchgehendes Beispiel ' heißt, dass wir nicht mit vielen verschiedenen kurzen Übungen arbeiten, sondern nach dem 1. Kapitel ,Grundlagen der Investitionsrechnung ' permanent anhand des genannten sehr umfangreichen Beispiels arbeiten werden.

Und ,konkret ' bedeutet, dass die Investition derzeit von einer , richtigen“ Firma in der Tat angedacht wird. Daher werden als Ausgangsbasis des großen Beispiels auch die Gewinn- und Verlustrechnung sowie die Bilanz dieser Gesellschaft dargestellt (Ist Zahlen Periode 1 und Planungen für Periode 2 und Periode 3) und am Ende des Buches die Auswirkungen auf das Zahlenwerk durch die Investition abgebildet.

Die gewählte Gesellschaft ist wieder die HTC - Heesen Top Cars. Diese Gesellschaft kennen manche von Ihnen eventuell schon und zwar von meinem Buch ,Bilanzplanung und Bilanzgestaltung - Fallorientierte Bilanzerstellung und Beratung $6 .{ }^{1}$ Dort zeige ich, ebenfalls an einem durchgehenden Excel basierten Fall (HTC), wie man Bilanzen und GuVs sukzessiv analysieren und mittels geeigneter und mathematisch logischer Planungsparameter auch leicht planen und wirklich intelligent und legal gestalten kann. ${ }^{2}$

Dieses Buch ,Investitionsrechnung für Praktiker" ist damit quasi der 2. Band in einer Reihe analytischer Werke für Praktiker mit dem immer identischen Unternehmen HTC Heesen Top Cars und den immer identischen Zahlen.

Die HTC, ein Automobilhändler, muss also eine Investition tätigen. Diese rechnen wir hier im Detail konkret durch und integrieren dann zum Schluss diese Investition in die Bilanzen und GuV der HTC, also quasi , Vorher ${ }^{6}$ und ,Nachher ${ }^{6}$ in der Gegenüberstellung.

Sie werden sehen, auch wenn manchmal die Optik bezüglich einer Formel eher abschreckt, es wird Spaß machen, umso mehr, je tiefer man in die Materie eindringt und sie verinnerlicht.

\footnotetext{
${ }^{1}$ Ebenfalls erschienen im Gabler Verlag, Wiesbaden, 2012: Bernd Heesen: Bilanzplanung und Bilanzgestaltung - Fallorientierte Bilanzerstellung und Beratung, 3. Auf. 2012, ISBN 978-3-83494024-7, 404 Seiten.

${ }^{2}$ Auch diese Excel Tools können Sie sich unter www.springer.com oder www.ifak-bgl.com kostenfrei in ebenfalls 2 Versionen (fertig und als Übungsdatei) auf Ihre Rechner laden.
} 


\section{Inhaltsverzeichnis}

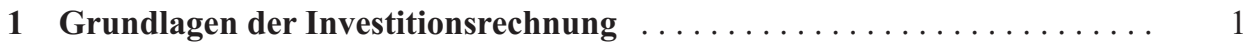

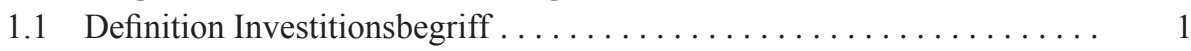

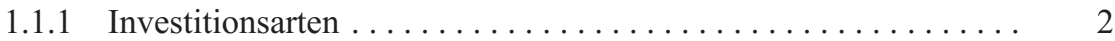

1.2 Investitionsprozess . . . . . . . . . . . . . . . . . . 3

1.3 Klassische Investitionsrechnungsverfahren $\ldots \ldots \ldots \ldots \ldots \ldots \ldots \ldots$

1.3.1 Übersicht Investitionsrechenverfahren $\ldots \ldots \ldots \ldots \ldots \ldots \ldots . \ldots$

1.3.2 Statische Investitionsrechenverfahren ............... 6

1.3.3 Genereller Aussagewert statischer Verfahren ... . . . . . . . . 15

1.3.4 Dynamische Investitionsrechenverfahren . . . . . . . . . . . 15

2 Investitionsrechnung in der Praxis - Die große Fallstudie Teil I . . . . . 73

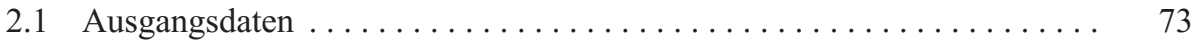

2.1.1 Die investierende Gesellschaft HTC - Heesen Top

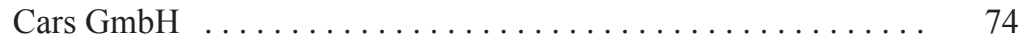

2.1.2 Die anstehende Investition . . . . . . . . . . . . . 80

2.1.3 Die Investition in der Deckungsbeitragsrechnung . . . . . . . . . 84

2.2 Investitionen wirklich richtig rechnen $\ldots \ldots \ldots \ldots \ldots \ldots \ldots \ldots$

2.2.1 Richtige Daten als Investitionsparameter $\ldots \ldots \ldots \ldots \ldots \ldots$

2.2.2 Dynamische Investitionsrechnungen . . . . . . . . . . . . . . . . 90

2.3 Richtige Rechnungen, aber falsche Daten . . . . . . . . . . . . . . . 104

2.3.1 Falsche Daten als Investitionsparameter . . . . . . . . . . . . . . . 104

2.3.2 Korrekte Dynamische Investitionsrechnungen mit

2.4 Unser Weg bis hier . . . . . . . . . . . . . . . . . . . . . 119

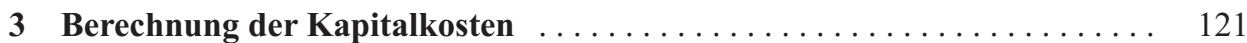

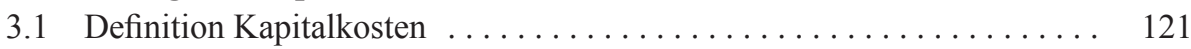

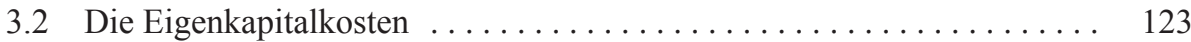

3.2.1 Risikofreier Satz und Risikprämie . . . . . . . . . . . . 123

3.2 .2 Der $\beta$-Faktor . . . . . . . . . . . . . . . . . . 127

3.2.3 Das CAPM - Capital Asset Pricing Model . . . . . . . . . . . . . . 129 
3.2.4 Leverage Betrachtungen und Integration . . . . . . . . . . 130

3.2.5 Die Eigenkapitalkosten aus steuerlicher Sicht . . . . . . . . . 133

3.3 Die Fremdkapitalkosten . . . . . . . . . . . . . . . . . . . . . . . . . . . . . . 134

3.4 Die Gesamtkapitalkosten - ,WACC ${ }^{6} \ldots \ldots \ldots \ldots \ldots \ldots \ldots \ldots \ldots \ldots$

3.5 Die ,Leverage ' Berechnung und Integration nach ,Stewart' . . . . . . . . . 138

3.5.1 Eigenkapitalkosten nach, Stewart ${ }^{\star} \ldots \ldots \ldots \ldots \ldots \ldots \ldots \ldots \ldots$

3.5.2 Fremdkapitalkosten nach ,Stewart' . . . . . . . . . . . . . 140

3.5.3 Gesamtkapitalkosten nach, Stewart ${ }^{\star} \ldots \ldots \ldots \ldots \ldots \ldots \ldots \ldots 14$

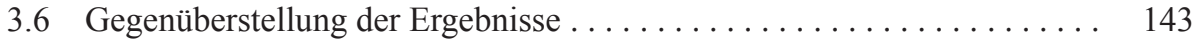

3.7 Zusammenfassung . . . . . . . . . . . . . . . . . . . . . . . . . . 145

3.8 Grafische Darstellungen . . . . . . . . . . . . . . . . . . . . 149

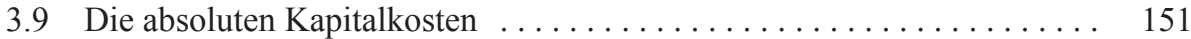

3.10 Mathematische Ableitung von ß-Faktoren (,De- und Relevern') . . . . 156

3.11 Abschließende Zusammenfassung und Auswirkungen auf

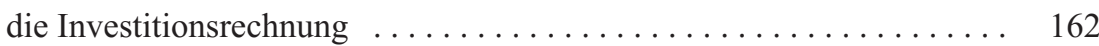

4 Die Investitionsergebnisse in der Gewinn- und Verlustrechnung . . . . . 167

4.1 Die (unzureichende) Bankenpräsentation . . . . . . . . . . . . . . 167

$4.2 \mathrm{GuV}$ und Bilanz als Grundlage von Bankenentscheidungen . . . . . . . . . 170

4.3 (Fehlende) Posten in der HTC Investitions GuV . . . . . . . . . . . . 173

4.3.1 Umsatzerlöse . . . . . . . . . . . . . . . . . . . . . 173

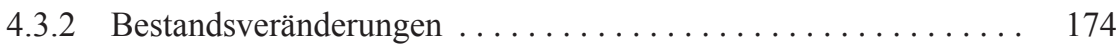

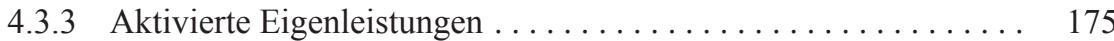

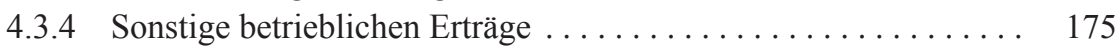

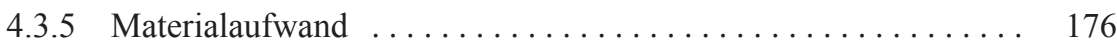

4.3.6 Personalkosten . . . . . . . . . . . . . . . . . . . . 176

4.3 .7 Abschreibungen ... . . . . . . . . . . . . . . . . . 177

4.3.8 Sonstige betriebliche Aufwendungen . . . . . . . . . . . . 178

4.3 .9 EBITD . . . . . . . . . . . . . . . . . . . 178

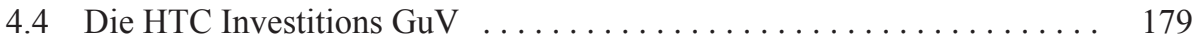

4.4 .1 Abschreibungen ............................ 179

4.4 .2 Betriebsergebnis . . . . . . . . . . . . . . . . . . . . 181

4.4.3 Fremdkapitalaufwendungen (Zinsen) . . . . . . . . . . . . . 182

4.4.4 EGT - Ergebnis der gewöhnlichen Geschäftstätigkeit . . . . . . . 184

4.4.5 Außerordentliches Ergebnis . . . . . . . . . . . . . . . . . 185

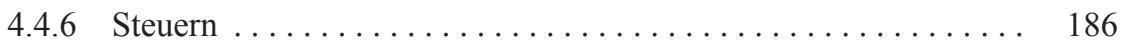

4.4 .7 Cash Flow . . . . . . . . . . . . . . . . . . . . . . . . . . . 188

4.5 Der HTC Vermögensnachweis . . . . . . . . . . . . . . . . . . . . . 189

4.6 Der falsche Investitionskostenansatz in der $\mathrm{GuV}$. . . . . . . . . . . . . 190

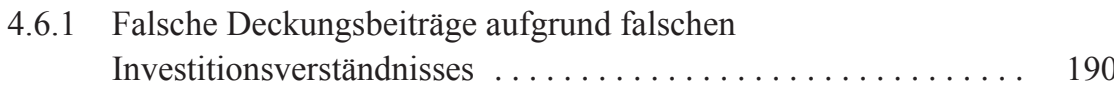

4.6.2 Richtige GuV aufgrund sauberer Überleitungen . . . . . . . . . . . 191

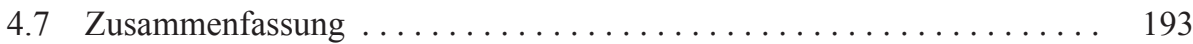


5 Auslandsinvestitionen und Vergleiche mit Investitionen im Inland . . . . . 199

5.1 Die Problematik bei Auslandsinvestitionen . . . . . . . . . . . . . . . . . . 199

5.2 Beispielhafte Darstellung anhand des HTC Investments . . . . . . . . . 201

5.3 Trennung der operativen und steuerlich geprägten Einflüsse . . . . . . . 205

5.4 Operative und steuerlich geprägte Einflüsse im Vergleich . . . . . . . . . 210

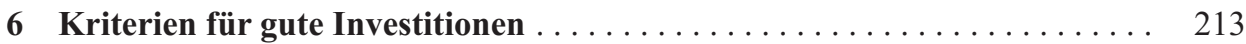

6.1 Tilgungen und eventuell Rückzahlung von Eigenkapital . . . . . . . 213

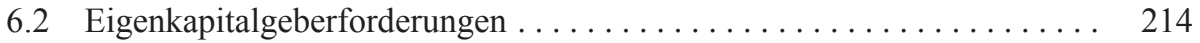

6.3 Neuinvestitionen . . . . . . . . . . . . . . . . . . . . . . . . . 215

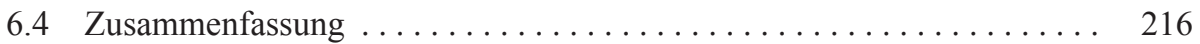

6.5 Integration fehlender Belastungen in die GuV Ergebnisse . . . . . . . . 217

6.5 .1 Tilgungen .......................... 219

6.5 .2 Eigenkapitalrückzahlungen ................... 219

6.5 .3 Dividenden ......................... 220

7 Angelsächsische Sichtweisen - Die große Fallstudie Teil II $\ldots \ldots \ldots \ldots \ldots$

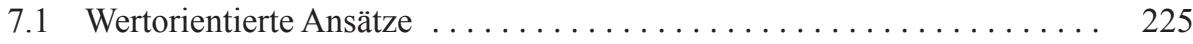

7.2 Ziele und Nutzen der Wertorientierung . . . . . . . . . . . . . 228

7.3 Ansätze der Wertorientierung in der Investitionsrechnung . . . . . . . . . 229

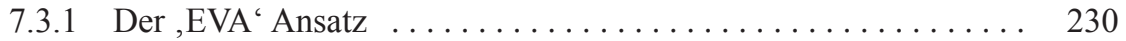

7.3 .2 Executive Summary - Teil I . . . . . . . . . . . . . . . . 242

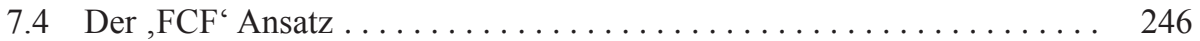

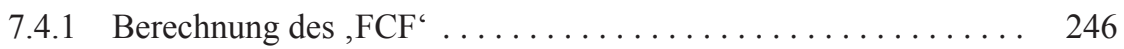

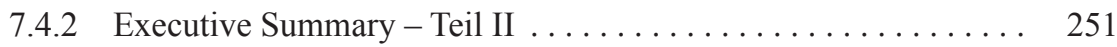

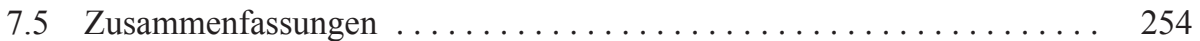

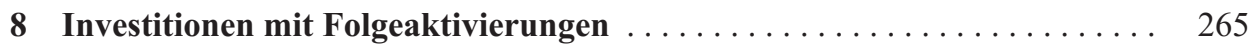

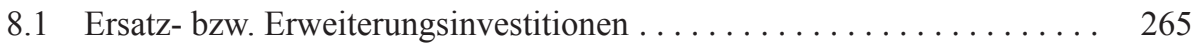

8.2 Ausgangsdatenlage . . . . . . . . . . . . . . . . . . . . 267

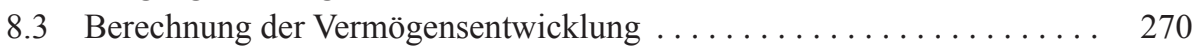

8.4 Berechnung der Kapitalkosten . . . . . . . . . . . . . . . . . . . . . . . . 272

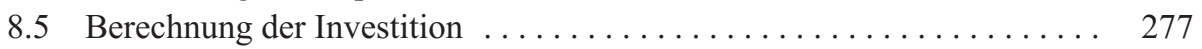

8.6 Grafische Darstellung der Ergebnisse . . . . . . . . . . . . . . . . . . . . 295

8.7 Weitergehende Auswertungen und Analysen . . . . . . . . . . . . . . . 296

8.8 QIKV . . . . . . . . . . 303

8.9 Die Gewinn und Verlustrechnung bei weiteren Aktivierungen . . . . . . . 305

8.9.1 Die Basis für die Betrachtungen gegen unendlich . . . . . . . . 311

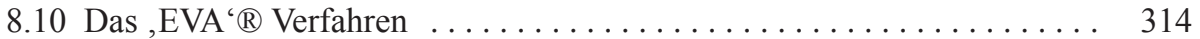

8.10 .1 Multiplikativer Ansatz . . . . . . . . . . . . . . . . 314

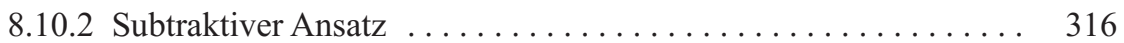




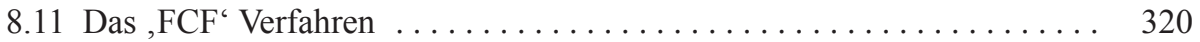

8.12 Ewige Rente mit Wachstum . . . . . . . . . . . . . . . . . . 324

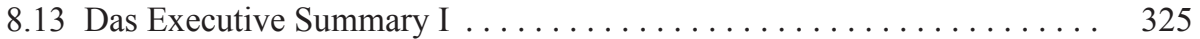

8.14 Das Executive Summary II . . . . . . . . . . . . . . . . . . . 339

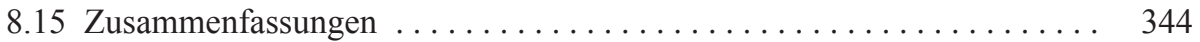

9 Auswirkungen der Investition auf die HTC GuV bzw. Bilanz . . . . . . . . 349

9.1 Die Investititionsdaten für die $\mathrm{HTC} \mathrm{GuV} \mathrm{\ldots \ldots \ldots \ldots ........} 350$

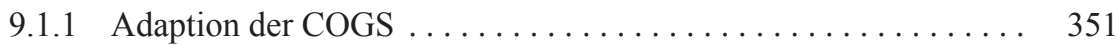

9.1.2 Integration der weiteren Investitionsdaten . . . . . . . . . 351

9.2 Die HTC GuV vor und nach Investition $\ldots \ldots \ldots \ldots \ldots \ldots \ldots \ldots . \ldots \ldots 2$

9.3 Die HTC Bilanz vor und nach Investition . . . . . . . . . . . . . . . . . 355

9.4 Analyse der Veränderungen durch die Investition . . . . . . . . . . . 358

9.4.1 Vermögen und Vermögensstruktur . . . . . . . . . . . . . . . . 359

9.4 .2 Kapital und Kapitalstruktur . . . . . . . . . . . . . . . . . . . . 362

9.4.3 Liquidität, Cash Flow bzw. Investitionspolitik . . . . . . . . . . 364

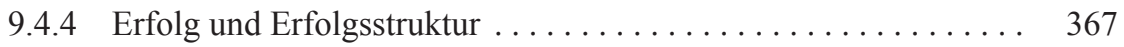

9.4 .5 Renditen .............................. 369

9.4.6 Sonstige Kennzahlen . . . . . . . . . . . . . . . . . 371

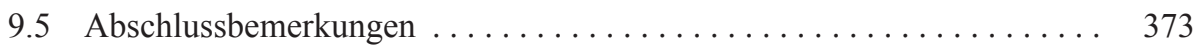

10 Das Wesentliche . . . . . . . . . . . . . . . . . . . . . . . 375

10.1 Klassische Investitionsrechnungen $\ldots \ldots \ldots \ldots \ldots \ldots \ldots \ldots \ldots \ldots$

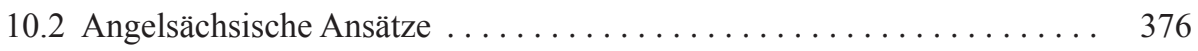

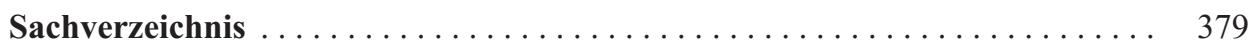

